\title{
A Novel Passive Dynamic Filtering Method of Composite Electrical Load
}

\author{
Weiping Fu ${ }^{a}$, Youxin Yuan ${ }^{b}$, Jing Chen ${ }^{c}$, Qijian Chen ${ }^{d}$ \\ School of Automation, Wuhan University of Technology, Wuhan, 430070, China \\ aemail: wsfwp@sina.cn, bemail: yyx2000@263.net, 'email:jingchen680@163.com
}

Keywords: Harmonics; Electrical System; Dynamic; Filter

\begin{abstract}
The use of non-linear and time-varying electronic devices, such as rectifiers and inverters, makes the current waveform of the grid seriously distorted. The harmonics generated by those devices have bad effects on the generation and transmission of electric energy. To deal with these problems, a novel passive dynamic filtering method is proposed in this paper. The method is based on the passive filter techniques proposed in 1950s. And it combines the conventional passive filtering techniques with modern computer technologies. When this method is applied in the electrical system, the harmonics can significantly be decreased. The following works have been done in the study: topology of the passive dynamic filter; principle of the passive dynamic filtering method; control system of the passive dynamic filter; implementation of the passive dynamic filtering method.
\end{abstract}

\section{Introduction}

With the rapid development of the national economy, the number of non-linear loads and devices used in various industries is growing [1]. Meanwhile, the harmonics generated by nonlinear loads and equipment are also increased in power grid. Non-linear loads, which are used in industrial and commercial fields, are the main source of harmonics. Therefore, the problem how to deal with the harmonics in the power system has attracted great attention among academia, industry and governments.

Though, passive power filters are advocated, such as LC filters. They are composed of some capacitors and reactors. But only some specific order harmonics can be eliminated by fixed capacitors and unchanged reactors. When parameters of the grid change, they cannot work well.

Therefore, a novel passive dynamic filtering method of the composite electrical load is proposed in this paper. The following works have been done in the study: topology of the passive dynamic filter; principle of the passive dynamic filtering method; control system of the passive dynamic filter; implementation of the passive dynamic filtering method.

\section{Principle of Passive Dynamic Filtering Method of Composite Electrical Load}

Nowadays the passive filters are widely used in China and abroad. The principle of the passive filter is to build a filter circuit with capacitors and reactors. The size of the capacitance and the inductance is properly adjusted, and then a circuit whose impedance is zero can be formed to filter specific order harmonic. For example, there is a kind of reactor whose impedance can be smoothly changed [2]. This kind of reactor has two winds. By properly changing the second winds, the value of impedance of the first wind can be adjusted. But in terms of harmonic filter, the harmonics cannot be filtered dynamically without the capacitors. So the adjustable reactors and the capacitors should be coordinately controlled to filter the harmonics. So in this paper, a passive dynamic filtering method of composite electrical load is proposed.

By analyzing the harmonic character of composite electrical load, this method can coordinate the capacitors and the reactors to reach the best filtering effect. Specifically: when the amount of harmonics in the actual operation of the grid changes, the intelligent controller can dynamically adjust the adjustable capacitors to a proper volume to absorb the grid harmonics. When the capacitance of the adjustable capacitors happens to change, the intelligent controller can adjust the impedance of the adjustable reactors. 


\section{Topological Structure of Passive Dynamic Filter}

According to the principle of the dynamic harmonic filtering method of the composite electrical load, the structure can be designed [3] [4]. The harmonic filtering is characterized by fastness, dynamic and smooth.

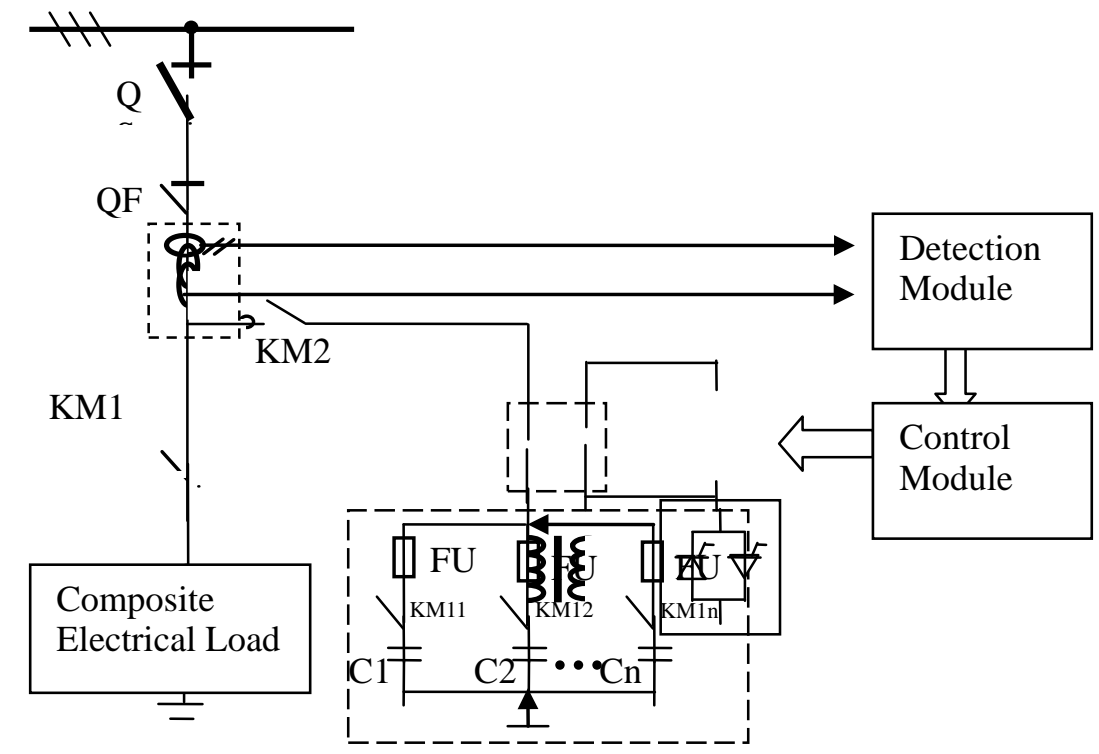

Fig .1. The Schemaric Diagrum of the Passive Dynamic Filtering Method

The passive dynamic filter consists of the following three units. They are the capacitor banks, the inductance converter, and control system. The topology of the passive dynamic filter is shown in Fig. 1.

In Fig.1, QS is the three-phase high voltage isolation switch; QF is the three-phase high voltage breaker; KM1 and KM2 are high voltage contactors. The capacitor bank is consists of some capacitors. By collecting the three phase voltage and current through the voltage sensor and current sensor which are shown as two arrows in Fig 1, the harmonics can be calculated by the detection module. And the information can be transmitted to the control module by using the ModBus protocol. The reactors and the capacitor bank are controlled by the control module.

\section{Implementation of Passive Dynamic Filtering Method}

Essential operation steps of the passive dynamic filter can be described as follows:

(1)Start running: Turn on the power source of the filter. And the intelligent controller starts to work. The high-voltage breaker QS and QF are switched on manually.

(2)The high voltage cont actor KM1 and KM2 are switched on under the control of the intelligent controller. The filter begins to operate.

(3)When the composite electrical load begins to work, contractors is changed by the intelligent controller from KM11 to KM1n to make the most proper capacitance connected to the grid after the analysis of the harmonic information. And then by receiving the pulse signal from the controller, the adjustable reactor can get a proper inductance. Under the harmonic frequency $f$, a low impedance path can be constituted by these control steps to absorb the harmonics.

(4) After the composite electrical load is under proper operation, the harmonics of the load is detected by the filtering system. When the load happens to change, the proper inductance and capacitance can be adjusted by the controller to maintain the resonant frequency.

After the above steps, the normal operation of the composite electrical load and harmonic filter can be achieved.

\section{Conclusion}

The method proposed in this paper is an optimization technique based on passive filtering techniques that both of the capacitance and the inductance of the filter can be changed [5]. Based on the harmonic characteristics generated by the composite electrical load, and by using the filtering 
techniques base on changeable reactors, this method can filter the harmonics generated by power supply system or the composite electrical load.

Therefore, compared with the existing filtering techniques, this filtering technique has following advantages:

(1)According to the harmonic frequency, this filter can dynamically search and track the harmonic source to filter the harmonics generated by electrical source or load. And intelligent controller can maintain grid resonant state by dynamically adjusting the inductance and the capacitance.

(2)When the capacitance of the capacitor bank happens to change, the intelligent controller can also adjust the inductance of the changeable reactor dynamically. So the intelligent controller can maintain the resonant state and guaranteed the filtering effect.

(3)When the harmonics generated by composite electrical load changes, the intelligent controller can calculate the most proper capacitance and control the contactors to get that capacitance. So the filter can get the proper volume to absorb the most harmonics. Then the intelligent controller controls the adjustable reactor to tune the resonance frequency.

\section{Acknowledgement}

This work is financially supported by the projects of Hubei Science and Technology Plans of China (Granted No: 2013BAA009).

\section{References}

[1] SINGH B, AL-HADDAD K, CHANDRA A. A review of active filters for power quality improvement [J]. IEEE Trans Ind Electron, 199946 (5) 960-971.

[2] Youxin Yuan, Yiping Xiao. Research on Impedance Conversion Mechanism of Variable Reactor [J]. Journal of Wuhan University of Technology, 20083 (30) 133-136.

[3] Haiping Lin, Jing chen, Youxin Yuan, Ming Chen, Zhou Chen, Simulation Research on Passive Dynamic Harmonic Filter [J]. Journal of Wuhan University of Technology, 201335 (3) 316-319.

[4] HU Zhi-kun, CHEN Zhi-wen, A novel digital control strategy of three-phase shunt active power filter under non-ideal mains voltages [J]. Journal of Central South University of Technology, 2011 (18) 2021-2030

[5] VU MINH QUANG, Han Wei. A New Type of PWM Rectifier with Function of Harmonic Suppression and Reactive Power Compensation. 25th Chinese Control and Decision Conference, 2013 